PROCEEDINGS OF THE

AMERICAN MATHEMATICAL SOCIETY

Volume 128, Number 1, Pages 261-267

S 0002-9939(99)05058-3

Article electronically published on May 6, 1999

\title{
ON CENTRAL LIMIT THEOREMS FOR SHRUNKEN RANDOM VARIABLES
}

\author{
ELIZABETH HOUSWORTH AND QI-MAN SHAO
}

(Communicated by Wei Y. Loh)

\begin{abstract}
We discuss Central Limit Theorems and absence of limiting distributions for shrunken random variables.
\end{abstract}

\section{INTRODUCTION}

For $r>0$ let $U_{r}$ be the shrinking operator: $U_{r}(x)=\max (|x|-r, 0) \operatorname{sgn}(x)$, or, equivalently,

$$
U_{r}(x)= \begin{cases}x+r & \text { for } \quad-\infty<x<-r, \\ 0 & \text { for } \quad-r \leq x \leq r \\ x-r & \text { for } \quad r<x<\infty\end{cases}
$$

This non-linear operator was studied by Jurek [2], [3] in relation to his work with s-self-decomposable distributions. Jurek showed that s-self-decomposable distributions were the limiting distributions of sums of the form

$$
U_{r_{n}}\left(X_{1}\right)+U_{r_{n}}\left(X_{2}\right)+\cdots+U_{r_{n}}\left(X_{n}\right)+x_{n}
$$

where $X_{1}, X_{2}, \ldots$ are independent random variables. Such distributions were also studied in a different context in [4] and [5].

If we let $\mathcal{G}$ be the class of Gaussian distributions, $\mathcal{S}$ the class of stable distributions, $\mathcal{L}$ the class of self-decomposable distributions, $\mathcal{U}$ the class of s-self-decomposable distributions, and $\mathcal{I D}$ the class of infinitely divisible distributions, then we have the following hierarchy: $\mathcal{G} \subset \mathcal{S} \subset \mathcal{L} \subset \mathcal{U} \subset \mathcal{I} D$. Thus the following question recently posed by Jurek naturally arises:

If $X, X_{1}, X_{2}, \ldots$ are independent and identically distributed (i.i.d.) random variables, what are the conditions on the distribution and the canonical form of $r_{n}$ so that there exist $x_{n}$ with (1.1) converging weakly to a standard normal distribution?

We have found that the condition on the distribution is that the tail must decay rapidly. We have:

Theorem 1. Let $X, X_{1}, X_{2}, \ldots$ be independent and identically distributed (i.i.d.) random variables. Let

$$
G(x)=\int_{0}^{\infty} t P(|X|>t+x) d t .
$$

Received by the editors March 25, 1998.

1991 Mathematics Subject Classification. Primary 60F05.

Key words and phrases. Central limit theorems, shrinking operator.

The first author's research was supported in part by the NSF under grant DMS9501611.

The second author's research was supported in part by the NSF under grant DMS9802451. 
If

(i) $0<G(x)<\infty$ for all $x>0$ and

(ii) $\lim _{x \rightarrow \infty} G(x+\epsilon) / G(x)=0$ for all $\epsilon>0$

then for any $r_{n} \rightarrow \infty$ satisfying

$$
\lim _{n \rightarrow \infty} 2 n G\left(r_{n}\right)=1
$$

and $x_{n}=-n E\left[\max \left(|X|-r_{n}, 0\right) \operatorname{sgn}(X)\right]$ we have

$$
U_{r_{n}}\left(X_{1}\right)+U_{r_{n}}\left(X_{2}\right)+\cdots+U_{r_{n}}\left(X_{n}\right)+x_{n} \Rightarrow N(0,1)
$$

where $\Rightarrow$ denotes weak convergence.

Remark 1 . It is easy to see that $G(x)<\infty$ for some $x>0$ if and only if $E X^{2}<\infty$. When $E X^{2}<\infty, G(x)$ is a non-increasing and continuous function. Therefore the solution $r_{n}$ to (1.3) exists. Moreover, $r_{n}$ must tend to $\infty$ under condition (i).

Remark 2. For $X$ with bounded support, one can use similar techniques as given below to show that the conclusion to Theorem 1 remains valid although one has to modify the choice of $r_{n}$ so that $n \operatorname{Var}\left(\max \left(|X|-r_{n}, 0\right) \operatorname{sgn}(X)\right) \rightarrow 1$.

It is easy to check that (ii) is satisfied for the normal distribution. However, many other standard distributions, such as the exponential distribution, do not satisfy (ii). On the other hand, as a partial converse, our second theorem shows that one cannot have a central limit theorem type result for (1.1) for such distributions:

Theorem 2. Let $X, X_{1}, X_{2}, \ldots$ be i.i.d. random variables. If there exists an $\varepsilon_{0}>0$ such that

$$
\liminf _{x \rightarrow \infty} P\left(|X|>x+\varepsilon_{0}\right) / P(|X|>x)>0,
$$

then there do not exist $r_{n} \rightarrow \infty$ and real numbers $x_{n}$ such that

$$
U_{r_{n}}\left(X_{1}\right)+U_{r_{n}}\left(X_{2}\right)+\cdots+U_{r_{n}}\left(X_{n}\right)+x_{n} \Rightarrow N(0,1) .
$$

Thus, for the Weibull family, with density functions:

$$
f(x ; a)=a x^{a-1} \exp \left(x^{-a}\right), x>0, a>0,
$$

we see that for $a>1$, (1.1) converges weakly to a normal distribution with the proper choice of $r_{n}$ given in Theorem 1, but no such Central Limit Theorem result holds for $0<a \leq 1$.

Our final result is that there is no non-degenerate limiting distribution for (1.1) for regular distributions:

Theorem 3. Let $X, X_{1}, X_{2}, \ldots$ be i.i.d. random variables with density function $f(x)$. Assume that there exist $p>1, c_{1} \geq 0, c_{2} \geq 0, c_{1}+c_{2}>0$ and a slowly varying function $l(x)($ at $\infty)$ such that

$$
\lim _{x \rightarrow+\infty} \frac{f(x)}{\left(l(x) / x^{p}\right)}=c_{1}, \quad \lim _{x \rightarrow+\infty} \frac{f(-x)}{\left(l(x) / x^{p}\right)}=c_{2} .
$$

Then for any $r_{n} \rightarrow \infty$ and real numbers $x_{n}, U_{r_{n}}\left(X_{1}\right)+U_{r_{n}}\left(X_{2}\right)+\cdots+U_{r_{n}}\left(X_{n}\right)+x_{n}$ does not have a non-degenerate limiting distribution. If $r_{n}$ satisfies

$$
\frac{n l\left(r_{n}\right)}{r_{n}^{p-1}} \rightarrow 0,
$$

then

$$
U_{r_{n}}\left(X_{1}\right)+U_{r_{n}}\left(X_{2}\right)+\cdots+U_{r_{n}}\left(X_{n}\right) \rightarrow 0 \text { in probability }
$$




\section{Proofs}

Throughout our proofs we make extensive use of the following theorem in [1], Theorem 18, p. 95. The statement of the theorem is modified slightly here to match our problem:

Theorem (A). Let $\left\{Y_{n}, Y_{n, 1}, Y_{n, 2}, \ldots, Y_{n, n}\right\}$ be a sequence of series of random variables i.i.d. within each series satisfying that for all $\varepsilon>0, P\left(Y_{n} \geq \epsilon\right) \rightarrow 0$. There will exist a sequence of real constants $x_{n}$ such that the distribution of the sums $\sum_{k=1}^{n} Y_{n k}+x_{n}$ converges weakly to the standard normal distribution if and only if the following conditions are satisfied:

$$
n P\left(\left|Y_{n}\right| \geq \varepsilon\right) \rightarrow 0 \text { for every } \varepsilon>0
$$

and

$$
n \operatorname{Var}\left(Y_{n} I_{\left\{\left|Y_{n}\right| \leq 1\right\}}\right) \rightarrow 1 .
$$

If these conditions are satisfied, then we may write

$$
x_{n}=-n E\left(Y_{n} I_{\left\{\left|Y_{n}\right| \leq 1\right\}}\right)+o(1) .
$$

Proof of Theorem 1. Let $Y_{n, k}=U_{r_{n}}\left(X_{k}\right)=\max \left(\left|X_{k}\right|-r_{n}, 0\right) \operatorname{sgn}\left(X_{k}\right)$ and $Y_{n}=$ $U_{r_{n}}(X)$. In order to show that we may choose our $x_{n}$ as given in Theorem 1 without truncating the tail as in Theorem A, we will show

$$
n E\left|Y_{n}\right| I_{\left\{\left|Y_{n}\right|>1\right\}} \rightarrow 0 .
$$

In order to show that the variance term (2.2) tends to 1 , we will show

$$
n E Y_{n}^{2} I_{\left\{\left|Y_{n}\right| \leq 1\right\}} \rightarrow 1
$$

and

$$
n\left(E\left|Y_{n}\right| I_{\left\{\left|Y_{n}\right| \leq 1\right\}}\right)^{2} \rightarrow 0 .
$$

First, in order to show (2.1), observe that $P\left(\left|Y_{n}\right|>\varepsilon\right)=P\left(|X|>r_{n}+\varepsilon\right)$. Since $P\left(|X|>r_{n}+\varepsilon\right) \leq P\left(|X|>t+r_{n}+\varepsilon / 2\right)$ for all $0 \leq t \leq \varepsilon / 2$, we see

$$
\begin{aligned}
P\left(|X|>r_{n}+\varepsilon\right) & \leq \frac{8}{\varepsilon^{2}} \int_{0}^{\varepsilon / 2} t P\left(|X|>t+r_{n}+\varepsilon / 2\right) d t \\
& \leq \frac{8}{\varepsilon^{2}} G\left(r_{n}+\varepsilon / 2\right) .
\end{aligned}
$$

Now (2.1) follows by our choice of $r_{n}$ and condition (ii).

To prove (2.3), we have

$$
\begin{aligned}
E\left|Y_{n}\right| I_{\left\{\left|Y_{n}\right|>1\right\}} & =E\left(|X|-r_{n}\right) I_{\left\{|X|>r_{n}+1\right\}} \\
& =P\left(|X|>r_{n}+1\right)+\int_{1 / 2}^{\infty} P\left(|X|>r_{n}+1 / 2+t\right) d t \\
& \leq P\left(|X|>r_{n}+1\right)+2 \int_{1 / 2}^{\infty} t P\left(|X|>r_{n}+1 / 2+t\right) d t \\
& \leq P\left(|X|>r_{n}+1\right)+2 G\left(r_{n}+1 / 2\right) .
\end{aligned}
$$

Note that $P\left(|X|>r_{n}+1\right) \leq 8 G\left(r_{n}+1 / 2\right)$ by the proof of (2.1) above with $\epsilon=1$. Thus, $E\left|Y_{n}\right| I_{\left\{\left|Y_{n}\right|>1\right\}} \leq 10 G\left(r_{n}+1 / 2\right)$ which yields (2.3) immediately by our choice of $r_{n}$ and condition (ii). 
To prove (2.4), let $G_{1}(x)=\int_{x}^{\infty} P(|X|>t) d t$. Note that

$$
\begin{aligned}
E Y_{n}^{2} I_{\left\{\left|Y_{n}\right| \leq 1\right\}}= & E\left(|X|-r_{n}\right)^{2} I_{\left\{r_{n}<|X| \leq r_{n}+1\right\}} \\
= & \int_{0}^{1} t^{2} d\left(-P\left(|X|>r_{n}+t\right)\right) \\
= & -P\left(|X|>1+r_{n}\right)+2 \int_{0}^{1} t P\left(|X|>r_{n}+t\right) d t \\
= & -P\left(|X|>1+r_{n}\right)+2 \int_{0}^{1} t d\left(-G_{1}\left(t+r_{n}\right)\right) \\
= & -P\left(|X|>1+r_{n}\right)-2 G_{1}\left(1+r_{n}\right) \\
& +2 \int_{0}^{1} \int_{t}^{\infty} P\left(|X|>s+r_{n}\right) d s d t \\
= & -P\left(|X|>1+r_{n}\right)-2 \int_{1+r_{n}}^{\infty} P(|X|>t) d t \\
& +2 \int_{0}^{1} \int_{t}^{1} P\left(|X|>s+r_{n}\right) d s d t+2 \int_{1}^{\infty} P\left(|X|>s+r_{n}\right) d s \\
= & -P\left(|X|>1+r_{n}\right)+2 \int_{0}^{1} s P\left(|X|>s+r_{n}\right) d s \\
= & -P\left(|X|>1+r_{n}\right)+2 G\left(r_{n}\right)-2 \int_{1}^{\infty} s P\left(|X|>s+r_{n}\right) d s .
\end{aligned}
$$

This proves (2.4) by (2.1), our choice of $r_{n}$, and by noting that

$$
\int_{1}^{\infty} s P\left(|X|>s+r_{n}\right) d s \leq \int_{1 / 2}^{\infty} 2 s P\left(|X|>s+r_{n}+1 / 2\right) d s \leq 2 G\left(r_{n}+1 / 2\right)
$$

so that $n \int_{1}^{\infty} s P\left(|X|>s+r_{n}\right) d s \rightarrow 0$.

Finally, we prove (2.5). It follows from the Hölder inequality that

$$
\begin{aligned}
n\left(E\left|Y_{n}\right| I_{\left\{\left|Y_{n}\right| \leq 1\right\}}\right)^{2} & =n\left(E\left(|X|-r_{n}\right) I_{\left\{r_{n}<|X| \leq 1+r_{n}\right\}}\right)^{2} \\
& \leq n P\left(r_{n}<|X| \leq 1+r_{n}\right) E\left(|X|-r_{n}\right)^{2} I_{\left\{r_{n}<|X| \leq 1+r_{n}\right\}} \rightarrow 0
\end{aligned}
$$

by (2.4) and the fact that $P\left(|X|>r_{n}\right) \rightarrow 0$ since $r_{n} \rightarrow \infty$.

This completes the proof of Theorem 1 .

Proof of Theorem 2. Since $r_{n} \rightarrow \infty$, we have

$$
\forall \varepsilon>0, \quad P\left(\max \left(|X|-r_{n}, 0\right)>\varepsilon\right) \rightarrow 0 .
$$

Assume that (1.2) converges weakly to a standard normal distribution. Then, by Theorem A again, we have

$$
\forall \varepsilon>0, n P\left(|X| \geq r_{n}+\varepsilon\right)=n P\left(\max \left(|X|-r_{n}, 0\right)>\varepsilon\right) \rightarrow 0
$$

and

$$
n \operatorname{Var}\left(\max \left(|X|-r_{n}, 0\right) \operatorname{sgn}(X) I_{\left\{\left|\max \left(|X|-r_{n}, 0\right) \operatorname{sgn}(X)\right|<1\right\}}\right) \rightarrow 1 .
$$

Thus, for sufficiently large $n$

$$
\begin{aligned}
1 / 2 & \leq n\left(E\left(|X|-r_{n}\right)^{2} I_{\left\{r_{n}<|X| \leq 1+r_{n}\right\}}\right) \\
& \leq n P\left(|X|>r_{n}\right),
\end{aligned}
$$


which together with (2.6) yields

$$
\forall \varepsilon>0, \quad \lim _{n \rightarrow \infty} P\left(|X|>r_{n}+\varepsilon\right) / P\left(|X|>r_{n}\right)=0 .
$$

This contradicts the assumption (1.4) and completes the proof of Theorem 2.

Proof of Theorem 3. Assume that (1.1) has a non-degenerate limiting distribution for some $r_{n} \rightarrow \infty$ and $\left\{x_{n}\right\}$. Let $Y_{n}=U_{r_{n}}(X)=\max \left(|X|-r_{n}, 0\right) \operatorname{sgn}(X)$. Then

$$
e^{i t x_{n}}\left(E e^{i t Y_{n}}\right)^{n} \rightarrow g(t),
$$

where $g(t)$ is a non-degenerate characteristic function. Write

$$
E e^{i t Y_{n}}=E \cos \left(t \max \left(|X|-r_{n}, 0\right)\right)+i E \sin \left(t \max \left(|X|-r_{n}, 0\right) \operatorname{sgn}(X)\right) .
$$

For $t \neq 0$, we have

$$
\begin{aligned}
& E \cos \left(t \max \left(|X|-r_{n}, 0\right)\right) \\
& \quad=1-P\left(|X|>r_{n}\right)+\int_{0}^{\infty} \cos (x) d\left(-P\left(|X|>r_{n}+x /|t|\right)\right) \\
& =1-\int_{0}^{\infty} \sin (x) P\left(|X|>r_{n}+x /|t|\right) d x .
\end{aligned}
$$

Put

$$
I_{n}(t)=\int_{0}^{\infty} \sin (x) P\left(|X|>r_{n}+x /|t|\right) d x .
$$

Using the properties of regular distributions, one can check that $I_{n}(t)$ is equal to

$$
\begin{aligned}
\sum_{k=0}^{\infty} & \left\{\int_{2 k \pi}^{(2 k+1) \pi} \sin (x) P\left(|X|>r_{n}+x /|t|\right) d x+\int_{(2 k+1) \pi}^{(2 k+2) \pi} \sin (x) P\left(|X|>r_{n}+x /|t|\right) d x\right\} \\
& =\sum_{k=0}^{\infty} \int_{0}^{\pi} \sin (x)\left\{P\left(|X|>r_{n}+(2 k \pi+x) /|t|\right)\right. \\
& =\int_{0}^{\pi} \sin (x)\left\{\sum_{k=0}^{\infty} P\left((2 k \pi+x) /|t|+r_{n}<|X| \leq r_{n}+((2 k+1) \pi+x) /|t|\right)\right\} d x \\
& \sim \int_{0}^{\pi} \sin (x)\left\{\sum_{k=0}^{\infty} \frac{\left(\left(c_{1}+c_{2}\right) \pi /|t|\right) l\left(r_{n}+(2 k \pi+x) /|t|\right)}{\left(r_{n}+(2 k \pi+x) /|t|\right)^{p}}\right\} d x \quad[\mathrm{by}(1.5)] \\
& \sim\left\{\sum_{k=0}^{\infty} \frac{\left(\left(c_{1}+c_{2}\right) \pi /|t|\right) l\left(r_{n}+2 k \pi /|t|\right)}{\left(r_{n}+2 k \pi /|t|\right)^{p}}\right\} \int_{0}^{\pi} \sin (x) d x \\
& \sim 2\left(c_{1}+c_{2}\right)(\pi /|t|) \int_{0}^{\infty} \frac{l\left(r_{n}+2 x \pi /|t|\right)}{\left(r_{n}+2 x \pi /|t|\right)^{p}} d x \\
& =\left(c_{1}+c_{2}\right) \int_{0}^{\infty} \frac{l\left(r_{n}+x\right)}{\left(r_{n}+x\right)^{p}} d x \\
& \sim \frac{\left(c_{1}+c_{2}\right) l\left(r_{n}\right)}{(p-1) r_{n}^{p-1}} .
\end{aligned}
$$


Therefore

$$
E \cos \left(t \max \left(|X|-r_{n}, 0\right)\right)-1 \sim-\frac{\left(c_{1}+c_{2}\right) l\left(r_{n}\right)}{(p-1) r_{n}^{p-1}} .
$$

To estimate $E \sin \left(t \max \left(|X|-r_{n}, 0\right) \operatorname{sgn}(X)\right)$, write $E \sin \left(t \max \left(|X|-r_{n}, 0\right) \operatorname{sgn}(X)\right)$

$=E \sin \left(t\left(X-r_{n}\right)\right) I_{\left\{X>r_{n}\right\}}-E \sin \left(t\left(-X-r_{n}\right)\right) I_{\left\{-X>r_{n}\right\}}$.

Following the proof of (2.8), we have

$$
\begin{aligned}
E & \sin \left(t\left(|X|-r_{n}\right) I_{\left\{X>r_{n}\right\}}\right)=\int_{0}^{\infty} \sin (t x) d\left(-P\left(X>r_{n}+x\right)\right) \\
= & t \int_{0}^{\infty} \cos (t x) P\left(X>r_{n}+x\right) d x \\
= & (t /|t|) \int_{0}^{\infty} \cos (x) P\left(X>r_{n}+x /|t|\right) d x \\
= & \operatorname{sgn}(t) \int_{0}^{\pi / 2} \cos (x) P\left(X>r_{n}+x /|t|\right) d x \\
& +\operatorname{sgn}(t) \sum_{k=0}^{\infty}\left\{\int_{2 k \pi+\pi / 2}^{(2 k+1) \pi+\pi / 2} \cos (x) P\left(X>r_{n}+x /|t|\right) d x\right. \\
& \left.\quad+\int_{(2 k+1) \pi+\pi / 2}^{(2 k+2) \pi+\pi / 2} \cos (x) P\left(X>r_{n}+x /|t|\right) d x\right\} \\
= & \operatorname{sgn}(t) \int_{0}^{\pi / 2} \cos (x) P\left(X>r_{n}+x /|t|\right) d x \\
& +\operatorname{sgn}(t) \sum_{k=0}^{\infty}\left\{\int _ { 0 } ^ { \pi } \operatorname { s i n } ( x ) \left\{-P\left(X>r_{n}+(x+2 k \pi+\pi / 2) /|t|\right)\right.\right. \\
& \left.\left.+P\left(X>r_{n}+(x+(2 k+1) \pi+\pi / 2) /|t|\right)\right\} d x\right\} \\
\sim & -\frac{c_{1} \operatorname{sgn}(t) l\left(r_{n}\right)}{(p-1) r_{n}^{p-1}} . \\
& \operatorname{sgn}(t) \frac{c_{1} l\left(r_{n}\right)}{r_{n}^{p}} \int_{0}^{\pi / 2} \cos (x) d x \\
& -\left(c_{1} \pi /|t|\right) \operatorname{sgn}(t) \sum_{k=0}^{\infty} \frac{l\left(r_{n}+2 k \pi /|t|\right)}{\left(r_{n}+2 k \pi /|t|\right)^{p}} \int_{0}^{\pi} \sin (x) d x
\end{aligned}
$$

Similarly,

Hence

$$
E \sin \left(t\left(-X-r_{n}\right) I_{\left\{-X>r_{n}\right\}}\right) \sim-\frac{c_{2} \operatorname{sgn}(t) l\left(r_{n}\right)}{(p-1) r_{n}^{p-1}}
$$

$$
E \sin \left(t \max \left(|X|-r_{n}, 0\right) \operatorname{sgn}(X)\right) \sim\left(c_{2}-c_{1}\right) \operatorname{sgn}(t) \frac{l\left(r_{n}\right)}{(p-1) r_{n}^{p-1}} .
$$

From (2.8) and (2.9) it follows that

$$
\left(E e^{i t Y_{n}}\right)^{n}=\left(1-\frac{l\left(r_{n}\right)}{(p-1) r_{n}^{p-1}}\left(c_{1}+c_{2}+i \operatorname{sgn}(t)\left(c_{1}-c_{2}\right)+o(1)\right)\right)^{n} .
$$


Consider four different cases:

Case 1. When $\liminf _{n \rightarrow \infty} \frac{n l\left(r_{n}\right)}{r_{n}^{p-1}}<\limsup _{n \rightarrow \infty} \frac{n l\left(r_{n}\right)}{r_{n}^{p-1}},(2.7)$ contradicts (2.10).

Case 2. When $\lim _{n \rightarrow \infty} \frac{n l\left(r_{n}\right)}{r_{n}^{p-1}}=\infty,(2.10)$ implies that for every $t \neq 0$,

$$
\left(E e^{i t Y_{n}}\right)^{n} \rightarrow 0
$$

which is also in contradiction with (2.7).

Case 3. When $\lim _{n \rightarrow \infty} \frac{n l\left(r_{n}\right)}{r_{n}^{p-1}}=a$, where $0<a<\infty$, we obtain from (2.10) that

$$
\forall t \neq 0, \quad\left(E e^{i t Y_{n}}\right)^{n} \rightarrow \exp \left(-\frac{a}{p-1}\left(c_{1}+c_{2}+i \operatorname{sgn}(t)\left(c_{1}-c_{2}\right)\right)\right) .
$$

Since $\left(E e^{i 0 Y_{n}}\right)^{n} \equiv 1$, the limiting function of $\left(E e^{i t Y_{n}}\right)^{n}$ is not continuous, which contradicts the assumption that $g(t)$ is a characteristic function.

Case 4. When $\lim _{n \rightarrow \infty} \frac{n l\left(r_{n}\right)}{r_{n}^{p-1}}=0$, then we have

$$
\left(E e^{i t Y_{n}}\right)^{n} \rightarrow 1
$$

and hence the limiting distribution is degenerate.

This completes the proof of the theorem.

\section{ACKNOWLEDGEMENT}

The authors would like to thank the referee for his or her helpful comments and suggestions.

\section{REFERENCES}

[1] V. V. Petrov, Sums of Independent Random Variables, Springer-Verlag, New York, 1975. MR 52:9335

[2] Z. Jurek, Limit distributions for sums of shrunken random variables, Dissertationes Math. 185 (1981) PWN Warszawa. MR 82i:60018

[3] Z. Jurek, Relations between the s-selfdecomposable and selfdecomposable measures, Ann. Probab. 13 (1985), 592-608. MR 87a:60016

[4] P. Medgyessy, On a new class of unimodal infinitely divisible distribution functions and related topics, Studia Sci. Math. Hungar. 2 (1967), 441-446. MR 36:5979

[5] T. A. O'Connor, Infinitely divisible distributions with unimodal Levy spectral functions, Ann. Probab. 7 (1979), 494-499. MR 80b:60035

Department of Mathematics, University of Oregon, Eugene, Oregon 97403

E-mail address: eah@math.uoregon.edu

E-mail address: shao@math.uoregon.edu 